\title{
Heat stress resilient crops for future hotter environments
}

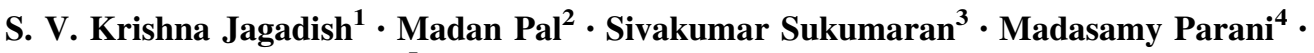 \\ Kadambot H. M. Siddique ${ }^{5}$
}

Published online: 12 December 2020

(C) Indian Society for Plant Physiology 2020

\begin{abstract}
The predicted increase in global mean temperature and its variability is a significant challenge for sustainable crop productivity under current and future climates. Research on understanding heat stress responses, identifying physiological processes, developing phenotyping protocols and unraveling molecular pathways that can help breed heat stress resilient crops is prominent in the crop science community. Research teams and laboratories contributing to this special issue title 'Heat Stress on Crop Growth and Development' have consolidated information on multiple dimensions of heat stress. The collection of reviews and research papers provides an excellent platform for developing future research objectives and is a valuable reference for students and researchers working on the heat stress response in crops.
\end{abstract}

Keywords Heat stress - Molecular mechanisms . Phenomics · Growth regulators · Candidate genes

S. V. Krishna Jagadish

kjagadish@ksu.edu

1 Department of Agronomy, Kansas State University, Manhattan, KS, USA

2 Division of Plant Physiology, ICAR-Indian Agricultural Research Institute, New Delhi 110012, India

3 Global Wheat Program, International Maize and Wheat Improvement Center (CIMMYT), Texcoco, Mexico

4 Genomics Laboratory, Department of Genetic Engineering, SRM Institute of Science and Technology, Kattankulathur, Tamil Nadu 603 203, India

5 The UWA Institute of Agriculture, The University of Western Australia (UWA), Perth, WA 6001, Australia

\section{Introduction}

Efforts to develop heat stress resilient crops are relatively recent compared to those for drought- and salt-tolerant crops. As a result of the industrial revolution in the 1800s and associated anthropogenic activities, such as the burning of coal, the rapid increase in atmospheric $\mathrm{CO}_{2}$, has increased global mean temperatures. According to an ongoing temperature analysis by scientists at NASA's Goddard Institute for Space Studies (GISS), Earth's average global temperature has increased by just over $1^{\circ} \mathrm{C}$ since 1880. Two-thirds of this warming has occurred since 1975 , at the rate of about $0.15-0.20{ }^{\circ} \mathrm{C}$ per decade (https://earth observatory.nasa.gov/world-of-change/global-temperatures). Current global climate models predict a further increases of 0.3 to $4.8^{\circ} \mathrm{C}$ (average $2.6^{\circ} \mathrm{C}$ ) by the end of the century (IPCC 2014). While climate change has a wide range of implications, the rapid increase in temperature is a primary factor effecting crop yields (Ortiz-Bobea et al. 2019). Under the current scenario about $75 \%$ of moderate daily hot extreme temperatures over land are attributed to human influence (Fischer and Knutti 2015). Historically, plants have adapted to a gradual increase in mean temperature. A similar increase in mean temperature in the future does not pose a threat to agricultural productivity, but it is the significant variability surrounding the mean, leading to heat shocks and heat waves that are perceived to derail efforts invested in sustaining global food production and security (Wheeler et al. 2000). Higher temperatures affect plant growth and development, with temperatures above critical thresholds inducing significant damage.

Field crops are highly sensitive to heat stress, more so during the reproductive stage than the vegetative stage (Ravikiran et al. 2020; Ostmeyer et al. 2020; Fig. 1). There has been considerable progress in understanding heat stress 
physiology from the whole plant to cellular level (Peer et al. 2020), advancing in phenomics to capture heat stress responses (Basavaraj and Rane 2020), deciphering epigenetic regulation at the molecular level (Shanker et al. 2020) and identifying promising candidates genes, such as glyoxalase (Garai et al. 2020), to address heat stress induced damage in crops. This special issue collated recent advances and current knowledge on heat stress responses in crops to provide a framework for designing objectives and hypotheses for further research in our quest to develop crop varieties that can sustain productivity under future hotter environments.

\section{Physiology, genetics, and breeding for enhancing heat tolerance in crops}

Many studies have demonstrated the negative impact of heat stress on the physiology of crop growth and development. Peer et al. (2020) extensively reviewed heat stress impacts on various physiological aspects, including water relations, membrane stability, respiration, and photosynthesis and indicated that they are negatively impacted while plant hormones, growth regulators, and metabolites are attuned at both the primary and secondary level. Plants respond to these modulations through the increased

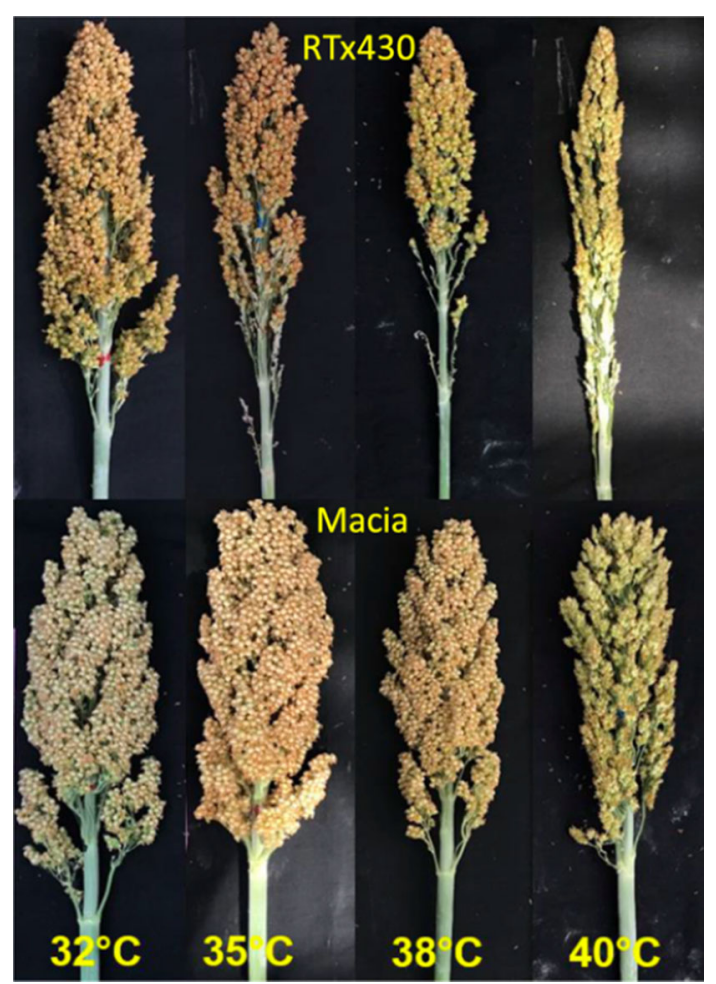

Fig. 1 Heat stress at flowering significantly reduced grain number in two sorghum genotypes (see Fig 5 b from Chiluwal et al. 2020). At 40 ${ }^{\circ} \mathrm{C}$, both genotypes were completely sterile, while Macia was more tolerant to $38{ }^{\circ} \mathrm{C}$ than $\mathrm{RT} x 430$ synthesis of heat shock proteins, reduced reactive oxygen species (ROS) production, membrane stability maintenance, osmoprotectant agglomeration, mitogen-activated protein kinase (MAPK) and calcium-dependent protein kinase (CDPK) cascade induction, antioxidant generation, and chaperone signaling, which eventually culminates in thermotolerance.

Impact of heat stress does not occur independently under field conditions but generally co-occurs with other stresses. This special issue comprises reviews that deal with heat and drought stress interactions. Sita and Kumar (2020) highlight the role of gamma amino butyric acid (GABA) in numerous metabolic processes, which partially safeguards plants from various abiotic stresses, including heat stress. GABA has a metabolic and signaling role in administering partial protection against heat stress by increasing leaf turgor, accumulating osmolytes, and reducing oxidative damage to plant membranes, and cellular homeostasis by stimulating antioxidants. Ostmeyer et al. (2020) reveal a positive role of nutrients in significantly ameliorating crop damage from drought stress but not heat stress. Nutrient interaction studies have proposed that the impact of drought stress on crops could be partially addressed through improved management options while that of heat stress can be achieved primarily by genetic improvement. Thomas et al. (2020) propose a phenotyping method for identifying rice genotypes with multiple abiotic stress tolerance during the seedling stage, primarily focusing on the root-shoot ratio as a screening tool.

Detailed climate change analysis has pointed to a rapid increase in night temperatures, impacting either a specific growth stage or the entire crop growth period. The influence of high night temperatures is not well understood as most studies impose combined higher day and night temperatures rather than night temperature only. Mamrutha et al. (2020) conclude that high night temperatures during grain filling is the most critical stage for reducing grain yield, harvest index, 1000-grain weight, and grain weight per spike. This study provides key traits for wheat breeders to enhance high night temperature tolerance in wheat.

Legumes are considered major sources of protein for the global human population (Foyer et al. 2016). Like cereals, legumes are equally sensitive to heat stress during the reproductive stage, leading to significant yield losses (Jha et al. 2020). Since heat stress is highly influenced by genotype $\mathrm{x}$ environment interactions, increasing the precision of phenotyping approaches would enable us to narrow the genotype-phenotype gap to understand the heat stress responses in legumes (Jha et al. 2020). Jha et al. (2020) propose a combination of next-generation breeding techniques, such as MAGIC, genomic selection, speed breeding, and genome editing tools, for accelerating the development of heat-tolerant cowpea genotypes that 
account for genotype $\mathrm{x}$ environment interactions. One approach to addressing heat stress induced yield losses is through the exogenous application of bioregulators (Kumar et al. 2020). In chickpea, the application of bioregulatorsABA (10 ppm), BA (40 ppm), SA (100 ppm), thiourea $(1000 \mathrm{ppm})$-activated the zeaxanthin pigment cycle prior to flowering, which had a favorable response to various physiological processes, including photosynthesis and related pigments, leading to a lower heat susceptibility index (Kumar et al. 2020a).

Rice and wheat are the major cereals that contribute significantly to global food security. Hybrid rice production is highly sensitive to high-temperature stress during panicle initiation and panicle development (Kumar et al. 2020b). Fertility reversibility is possible in rice CMS lines when the temperature exceeded $30{ }^{\circ} \mathrm{C}$ during the panicle initiation, a phenomenon captured by following a simple field-based technique (Kumar et al. 2020b). Further, Kumar et al. (2020b) demonstrate that temperature and daylength interactions play a role in the breakdown of sterility in rice CMS lines. Field based phenotyping during hot summer months identified novel heat-tolerant donors, such as NL44, which could be used to identify novel genomic regions (Ravikiran et al. 2020). The population derived from NL44 and PB 1 (Pusa Basmati 1) could be used to identify newer regions and additional molecular markers for stacking candidate genes/regions to further enhance heat tolerance in rice during the sensitive reproductive stages (Ravikiran et al. 2020). The judicious combination of conventional breeding and new technologies can help plants to efficiently cope with heat stress, including maintaining/enhancing yield to meet the nutritional requirements of the growing population (Verma et al. 2020).

\section{High-throughput phenotyping and genomic advances to minimize heat stress damage in crops}

Most studies on heat stress are conducted in pots in growth chambers or in greenhouses, which may not be readily applicable to field-based breeding efforts (Poorter et al. 2016). For the genetic dissection of heat stress tolerance, large-scale experiments are needed, and phenomics can play a major role. Trait-based breeding has always been a challenge as screening many genotypes for traits of interest is laborious and demands significant resources. Developing tools by capitalizing on advances in sensor technology will advance our capability of screening much larger numbers and diverse germplasm, which will significantly increase our chances of identifying novel donors for breeding increased heat tolerance in crops (Basavaraj and Rane 2020). Basavaraj and Rane (2020) provide extensive information on the need for and bottlenecks in implementing trait-based breeding and how advances in sensor-based high-throughput phenotyping could help in phenotyping large populations both rapidly and accurately for traits of interest.

\section{Integrating molecular components to understand heat stress responses}

Heat stress negatively impacts a wide range of processes including root system (physical support, water, and nutrient uptake), growth, flowering, pollination, seed set, grain filling, and seed yield. Increased ROS and dicarbonyl metabolite levels during heat stress lead to oxidative stress, which affects the normal biological functions of plants in multiple ways. Deploying ROS-scavenging enzymes and other enzymes that can detoxify dicarbonyl metabolites would be ideal for dealing with oxidative damage during heat stress. Methylglyoxal is a cytotoxic dicarbonyl metabolite, that can be detoxified by glyoxalase enzymes. While the role of glyoxalases under salinity, drought, and heavy metal stresses has been reviewed, research on their contribution to cope with heat stress has not received much attention. Garai et al. (2020) present an excellent review on the response of glyoxalases under heat stress and the extent to which these enzymes confer tolerance to heat stress. Perturbation in protein folding under heat stress leads to stress in the endoplasmic reticulum (ER). The unfolded protein response (URP), in the form of upregulation of chaperones and foldases and downregulation of secretory proteins, mitigated ER stress. Though URP is generally well studied, its role in heat stress is an emerging research area with great promise in developing thermotolerance. Malini et al. (2020) summarize the key findings related to this aspect; they also discuss the link between ER-associated degradation (ERAD) and plant growth regulators during heat stress. In addition to the direct role of genes, epigenetic modifications, such as methylation and histone modifications leading to altered gene regulation and memory of primary heat stress play a role in developing thermo tolerance to minimize the impact of subsequent heat stress events in the same or next generation. Also, epigenetic regulation of the heat stress response is mediated through miRNAs. Shanker et al. (2020) document transgenerational memory and gene expression regulation in response to heat through epigenetic modifications in histones and histone chaperones, chromatin remodeling, small RNA, and long noncoding RNAs. Their review also includes chromatin dynamics and heat stress-responsive epigenetic gene regulation. 


\section{Future directions}

A pressing priority is the need to develop reliable, rapid phenotyping tools and economically feasible facilities to capture the impact of heat stress under field conditions. Aspects such as high day and/or night temperatures and the interaction with vapor pressure deficit present a wide range of heat stress scenarios that would require well designed strategies to identify target traits for incorporating into breeding programs, depending on the target population of environments. A wide range of physiological, biochemical and molecular responses would require additional validation for their role in tolerating, escaping, or avoiding heat stress to help develop sensor-based high-throughput phenotyping, rapid lab-based assays, and breeder friendly markers, respectively, to develop a roadmap for the rapid development and deployment of heat-tolerant crop varieties. Going forward, advances in tools and protocols developed to integrate phenomics with genomics to enhance heat stress resilience in crops, should be validated for genotype $\mathrm{x}$ environment interactions and potential trade-offs that could negatively impact grain yield and/or quality under field conditions.

Acknowledgements SVKJ acknowledges contribution number 21-128-J from Kansas Agricultural Experiment Station.

\section{References}

Basavaraj, P. S., \& Rane, J. (2020). Avenues to realize potential of phenomics to accelerate crop breeding for heat tolerance. Plant Physiology Reports. https://doi.org/10.1007/s40502-020-005522.

Chiluwal, A., Bheemanahalli, R., Kanaganahalli, V., Boyle, Dan, Perumal, R., Pokharel, M., et al. (2020). Deterioration of ovary plays a key role in heat stress-induced spikelet sterility in sorghum. Plant Cell Environment, 43, 448-462.

Fischer, E., \& Knutti, R. (2015). Anthropogenic contribution to global occurrence of heavy-precipitation and high-temperature extremes. Nature Climate Change, 5, 560-564.

Foyer, C. H., Lam, H.-M., Nguyen, H. T., Siddique, K. H. M., Varshney, R., Colmer, T. D., et al. (2016). Neglecting legumes has compromised human health and sustainable food production. Nature Plants, 2, 16112. https://doi.org/10.1038/NPLANTS. 2016.112.

Garai, S., Bhowal, B., Pareek, A., Singla-Pareek, S. L., Kaur, C., \& Sopory, S. K. (2020). Expression dynamics of glyoxalase genes under high temperature stress in plants. Plant Physiology Reports. https://doi.org/10.1007/s40502-020-00545-1.

IPCC. (2014). Climate change 2014: synthesis report. In Core Writing Team, R. K. Pachauri, \& L. A. Meyer (Eds.), Contribution of working groups I, II and III to the Fifth Assessment report of the Intergovernmental Panel on Climate Change. Geneva, Switzerland: IPCC.

Jha, U. C., Nayyar, H., Jha, R., Paul, P. J., \& Siddique, K. H. M. (2020). Heat stress and cowpea: Genetics, breeding and modern tools for improving genetic gains. Plant Physiology Reports. https://doi.org/10.1007/s40502-020-00544-2.

Kumar, P., Yadav, S., \& Singh, M. P. (2020a). Bioregulators application improved heat tolerance and yield in chickpea (Cicer arietinum L.) by modulating zeaxanthin cycle. Plant Physiology Reports. https://doi.org/10.1007/s40502-020-00555-z.

Kumar, Y. L., Deka, S. D., Sarma, D., \& Gogoi, S. (2020b). Temperature stress and fertility reversibility in wild abortive CMS lines of rice for quality hybrid seed production. Plant Physiology Reports. https://doi.org/10.1007/s40502-020-00556$\mathrm{y}$.

Malini, M. K., Lekshmy, V. S., Pal, M., Chinnusamy, V., \& Kumar, M. N. (2020). Heat stress responses and the endoplasmic reticulum (ER) homeostasis in crop plants. Plant Physiology Reports. https://doi.org/10.1007/s40502-020-00548-y.

Mamrutha, H. M., Rinki, K., Venkatesh, K., Gopalareddy, K., Khan, H., Mishra, C. N., et al. (2020). Impact of high night temperature stress on different growth stages of wheat. Plant Physiology Reports. https://doi.org/10.1007/s40502-020-00558-w.

Ortiz-Bobea, A., Wang, H., Carrillo, C. M., \& Ault, T. R. (2019). Unpacking the climatic drivers of US agricultural yields. Environmental Research Letters, 14, 064003.

Ostmeyer, T., Parker, N., Jaenisch, B., Alkatomi, L., Bustamante, C., \& Jagadish, S. V. K. (2020). Impacts of heat, drought, and their interaction with nutrients on physiology, grain yield, and quality in field crops. Plant Physiology Reports. https://doi.org/10.1007/ s40502-020-00538-0.

Peer, L. A., Dar, Z. A., Lone, A. A., Bhat, M. Y., \& Ahamad, N. (2020). High temperature triggered plant responses from whole plant to cellular level: A review. Plant Physiology Reports. https://doi.org/10.1007/s40502-020-00551-3.

Poorter, H., Fiorani, F., Pieruschka, R., Wojciechowski, T., van der Putten, W. H., Kleyer, M., et al. (2016). Pampered inside, pestered outside? Differences and similarities between plants growing in controlled conditions and in the field. New Physiologist, 212(4), 838-855.

Ravikiran, K. T., Krishnan, S. G., Vinod, K. K., Dhawana, G., Dwivedia, P., Kumara, P., et al. (2020). A trait specific QTL survey identifies NL44, a NERICA cultivar as a novel source for reproductive stage heat stress tolerance in rice. Plant Physiology Reports. https://doi.org/10.1007/s40502-020-00547-z.

Shanker, A. K., Bhanu, D., \& Maheswari, M. (2020). Epigenetics and transgenerational memory in plants under heat stress. Plant Physiology Reports. https://doi.org/10.1007/s40502-020-00557$\mathrm{x}$.

Sita, K., \& Kumar, V. (2020). Role of gamma amino butyric acid (GABA) against abiotic stress tolerance in legumes: A review. Plant Physiology Reports. https://doi.org/10.1007/s40502-02000553-1.

Thomas, T., Purushothaman, J., Janarthanan, R., Anusuya, N., Medisetti, P. G., Karthick, J., et al. (2020). Identification of rice genotypes for seedling stage multiple abiotic stress tolerance. Plant Physiology Reports. https://doi.org/10.1007/s40502-02000549-x.

Verma, S., Kumar, N., Verma, A., Singh, H., Siddique, K. H. M., \& Singh, N. P. (2020). Novel approaches to reduce heat stress impact on crop growth and development. Plant Physiology Reports. https://doi.org/10.1007/s40502-020-00550-4.

Wheeler, T. R., Craufurd, P. Q., Ellis, R. H., Porter, J. R., \& Prasad, P. V. V. (2000). Temperature variability and the yield of annual crops. Agriculture, Ecosystems \& Environment, 82, 159-167.

Publisher's Note Springer Nature remains neutral with regard to jurisdictional claims in published maps and institutional affiliations. 